\title{
ARTICLE \\ Neurophysiological signature of gamma-hydroxybutyrate augmented sleep in male healthy volunteers may reflect biomimetic sleep enhancement: a randomized controlled trial
}

\author{
Dario A. Dornbierer ${ }^{1,2,3}$, Diego M. Baur ${ }^{1,4}$, Benjamin Stucky iD $^{1,4}$, Boris B. Quednow (D) $^{3}$, Thomas Kraemer ${ }^{2}$, Erich Seifritz ${ }^{3,4,5}$,
} Oliver G. Bosch (iD ${ }^{3}$ and Hans-Peter Landolt (iD ${ }^{1,4}$

\begin{abstract}
Gamma-hydroxybutyrate $(\mathrm{GHB})$ is an endogenous $\mathrm{GHB} / \mathrm{GABA}_{\mathrm{B}}$ receptor agonist, which has demonstrated potency in consolidating sleep and reducing excessive daytime sleepiness in narcolepsy. Little is known whether GHB's efficacy reflects the promotion of physiological sleep mechanisms and no study has investigated its sleep consolidating effects under low sleep pressure. GHB (50 mg/kg p.o.) and placebo were administered in 20 young male volunteers at 2:30 a.m., the time when GHB is typically given in narcolepsy, in a randomized, double-blinded, crossover manner. Drug effects on sleep architecture and electroencephalographic (EEG) sleep spectra were analyzed. In addition, current source density (CSD) analysis was employed to identify the effects of GHB on the brain electrical sources of neuronal oscillations. Moreover, lagged-phase synchronization (LPS) analysis was applied to quantify the functional connectivity among sleep-relevant brain regions. GHB prolonged slow-wave sleep (stage N3) at the cost of rapid eye movement (REM) sleep. Furthermore, it enhanced delta-theta $(0.5-8 \mathrm{~Hz})$ activity in NREM and REM sleep, while reducing activity in the spindle frequency range $(13-15 \mathrm{~Hz})$ in sleep stage N2. The increase in delta power predominated in medial prefrontal cortex, parahippocampal and fusiform gyri, and posterior cingulate cortex. Theta power was particularly increased in the prefrontal cortex and both temporal poles. Moreover, the brain areas that showed increased theta power after GHB also exhibited increased laggedphase synchronization among each other. Our study in healthy men revealed distinct similarities between GHB-augmented sleep and physiologically augmented sleep as seen in recovery sleep after prolonged wakefulness. The promotion of the sleep neurophysiological mechanisms by GHB may thus provide a rationale for GHB-induced sleep and waking quality in neuropsychiatric disorders beyond narcolepsy.
\end{abstract}

Neuropsychopharmacology (2019) 44:1985-1993; https://doi.org/10.1038/s41386-019-0382-z

\section{INTRODUCTION}

Sleep-wake disturbances are highly prevalent in society, especially among patients with neurological and psychiatric disorders [1-3]. The restoration and promotion of physiological sleep represents a core aim in psychopharmacology. Benzodiazepines and $z$-substances are positive allosteric $\mathrm{GABA}_{\mathrm{A}}$ receptor modulators, which are frequently used to manage sleep disturbances $[4,5]$. Yet, their clinical efficacy is often insufficient and these compounds do not promote physiological sleep $[6,7]$. Furthermore, these drugs often cause neurocognitive deficits $[4,8]$. Therapeutic alternatives are, thus, urgently needed.

Gamma-hydroxybutyrate (GHB or sodium oxybate), an endogenous $G H B / G A B A_{B}$ receptor agonist, has recently gained interest as a potential pharmacological agent to promote physiological sleep (for review, see [9]). When administered at bedtime, GHB enhances sleep efficiency and electroencephalographic (EEG) slow waves in non-rapid eye movement (NREM) sleep, and reduces pathological sleepiness in patients suffering from narcolepsy, Parkinson's disease, and fibromyalgia [10-15]. Given the hypothesized importance of EEG slow waves in NREM sleep for cognition and emotions [16, 17], GHB's beneficial effects have primarily been assigned to its ability to promote slow-wave sleep and to produce EEG slow oscillations during sleep [18]. Nevertheless, GHB also reduces cataplexies and hypnagogic hallucinations in narcolepsy, which may indicate that it normalizes REM sleep e.g., by reducing the number of awakenings during REM sleep episodes [19].

Despite the clinical observations summarized above, it is currently unclear whether GHB promotes physiological sleep mechanisms and enhances homeostatically regulated sleep intensity. Sleep homeostasis refers to the general biological principle that sleep is more intense than baseline sleep when recovering from prolonged waking or sleep restriction [20]. Sleep intensity can be reliably quantified in the EEG power spectrum during NREM sleep [21]. More specifically, it is well established that EEG delta $(\sim 0.5-4.5 \mathrm{~Hz})$ and theta $(\sim 6-9 \mathrm{~Hz})$ activity in NREM and REM sleep are enhanced over frontal brain sites after extended wakefulness, whereas activity in the frequency range

\footnotetext{
${ }^{1}$ Institute of Pharmacology and Toxicology, University of Zürich, Zürich, Switzerland; ${ }^{2}$ Department of Forensic Pharmacology and Toxicology, Zurich Institute of Forensic Medicine, University of Zürich, Zürich, Switzerland; ${ }^{3}$ Department of Psychiatry, Psychotherapy and Psychosomatics, Psychiatric Hospital, University of Zürich, Lenggstrasse 31,

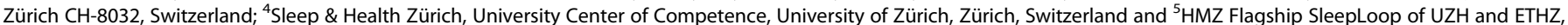
Zürich, Switzerland

Correspondence: Dario A. Dornbierer (dornbierer@pharma.uzh.ch)
}

Received: 13 November 2018 Revised: 25 March 2019 Accepted: 1 April 2019

Published online: 8 April 2019 
of sleep spindles $(\sim 12-15 \mathrm{~Hz})$ in NREM sleep is reduced $[22,23]$. Moreover, the duration of stage N2 and REM sleep are reduced at the cost of stage N3 sleep [24, 25]. The reverse pattern is observed in the second half of a sleep episode, reflecting the declining trend of sleep propensity and sleep intensity throughout the night.

Because of its short half-lifetime, GHB in clinical practice is typically administered in two doses: one dose at bedtime and one dose in the middle of the sleep episode. In most previous studies in healthy volunteers, GHB was administered at the beginning of the night when sleep intensity is high $[14,15]$. Here we administered a therapeutic dose $(50 \mathrm{mg} / \mathrm{kg})$ of GHB to 20 healthy young men only once in the middle of the night and employed complementary neurophysiological imaging methods to test the hypothesis that the drug-induced effects on sleep and the sleep EEG mimic physiologically enhanced sleep intensity. Thus, we elucidated in detail the neurophysiological signature of GHB-augmented sleep, by analyzing the drug effects on sleep architecture, sleep EEG spectra, brain electrical sources, and functional connectivity among sleep relevant brain regions. To evaluate the similarities and differences of GHBaugmented sleep and naturally enhanced sleep, we compared the present results with the neurophysiological changes that have been reported by us and others in recovery sleep after acute sleep deprivation.

\section{METHODS}

\section{Permission}

The study was approved by SwissMedic and Ethics Committee of the Canton of Zurich and registered at ClinicalTrials.gov (NCT02342366). All participants provided written informed consent according to the declaration of Helsinki.

\section{Study design}

The study followed a randomized, placebo-controlled, balanced, double-blind, crossover design. All study participants completed a screening night in the sleep laboratory, to exclude sleeprelated disorders such as sleep apnea ( $>5$ apneas/hypopneas per hour of sleep), restless legs syndrome ( $>5$ periodic limb movements per hour of sleep), occurrence of sleep onset REM sleep episodes, and insufficient sleep efficiency $(<80 \%)$ before definite enrollment into the study. The study protocol consisted of two randomized, experimental nights (GHB and placebo) separated by a washout phase of 7 days. Each experimental night was preceded by an adaptation night for habituation to the laboratory environment.

\section{Participants}

Twenty healthy, male volunteers (mean age: $25.8 \pm 5.1$ years) completed the study. Following criteria were required for inclusion: (i) male sex (to avoid unknown pregnancy and a potential impact of menstrual cycle on primary outcome variables); (ii) age within the range of $18-40$ years; (iii) absence of somatic or psychiatric disorders; (iv) no first-degree relatives with a history of heritable psychiatric disorders such as schizophrenia, bipolar disorder, autism, and attention deficit hyperactivity disorder; (v) non-smoker; (vi) no history of regular drug use (lifetime use $<5$ occasions of each drug, except occasional cannabis use). No participant reported previous experiences with GHB in their life. Participants had to refrain from illegal drugs for 2 weeks and from caffeine for 1 week before the first experimental night and throughout the study. No alcohol was allowed $24 \mathrm{~h}$ before each study night. Participants were instructed to keep a regular sleep-wake rhythm with $8 \mathrm{~h}$ in bed from 23:00 to 07:00 during 1 week before the first experimental night and in the week between the two experimental nights. To ensure compliance with this requirement, participants wore an actimeter on the nondominant arm and kept a sleep-wake diary. Both actigraphic recordings and sleep-wake diaries were qualitatively inspected by an experienced member of the study team before the experimental nights, to ensure adherence of the subject to the imposed sleep-wake schedule. No subject had to be excluded because of violation of the instructed sleep schedule. Moreover, sleep efficiency in all adaptation nights preceding each experimental night was above $80 \%$.

All participants received a monetary compensation for study participation.

\section{Urine immunoassay}

On each test night, urine samples were taken upon arrival in the laboratory, to ensure that all participants abstained from illegal drug use (Drug-Screen Multi 12-AE, Nal von Minden $\mathrm{GmbH}$, Regensburg, DE).

\section{Drug administration}

Study volunteers sleeping in the sleep laboratory were awoken at 2:30 a.m. to receive $50 \mathrm{mg} / \mathrm{kg}$ of GHB (Xyrem ${ }^{\circledR}$; Cantonal Pharmacy, Zurich, Switzerland) dissolved in $2 \mathrm{dl}$ of orange juice or placebo, matched in appearance and taste. The administered dose represents the maximal therapeutic starting dose in narcolepsy. After GHB/placebo intake, volunteers where allowed to immediately return to sleep.

\section{EEG data acquisition}

Sleep was quantified by all-night polysomnography with Rembrandt ${ }^{\circledR}$ Datalab (Version 8; Embla Systems, Planegg, Germany) from 23:00 (lights-off) to 07:00 (lights-on). The recording setup consisted of 19 EEG electrodes (Fp1, Fp2, F3, F4, F7, F8, Fz, T3, T4, T5, T6, C3, C4, Cz, P3, P4, Pz, O1, 02) according to the 10-20 system [26], a bipolar electrooculogram, a submental electromyogram (EMG), and an electrocardiogram (ECG). The individual EEG electrode coordinates were marked by cutting the subjects' hair at the electrode position, to ensure that the electrodes were placed at the very same place in both experimental conditions.

All data were recorded with dedicated polygraphic amplifiers (Artisan ${ }^{\circledR}$, Micromed, Mogliano Veneto, Italy). As in previous studies, the analog signals were conditioned by a high-pass filter (EEG: $-3 \mathrm{~dB}$ at $0.15 \mathrm{~Hz}$; EMG: $10 \mathrm{~Hz}$; ECG: $1 \mathrm{~Hz}$ ) and an antialiazing low-pass filter $(-3 \mathrm{~dB}$ at $67.2 \mathrm{~Hz})$, digitized and stored with a resolution of $256 \mathrm{~Hz}$ (sampling frequency of $256 \mathrm{~Hz}$ ) [27, 28].

\section{Sleep stage scoring}

For sleep scoring, the C3-A2 derivation was used. Sleep variables were visually scored based on $30 \mathrm{~s}$ epochs according to the criteria of the American Academy of Sleep Medicine [29]. Movement- and arousal-related artifacts were visually identified and excluded from analyses. The following sleep variables were computed: (i) duration of sleep stages (N1, N2, N3, and REM sleep, and wakefulness); (ii) duration of NREM sleep (time spent in stages $\mathrm{N} 2$ and N3); (iii) total sleep time (TST; time spent in N1, N2, N3, and REM sleep); (iv) time in bed (TIB; time between lights-off and lights-on); and (v) sleep efficiency index $(\mathrm{SEI}=[\mathrm{TST} / \mathrm{TIB}] \times 100)$. Sleep variables were computed for the first and second halves of the sleep episodes, as well as for the entire night.

\section{Spectral analysis}

Power spectra were computed by a Fast-Fourier transform based on $4 \mathrm{~s}$ epochs (Hanning window, linear detrending, $50 \%$ overlap), resulting in a frequency resolution of $0.25 \mathrm{~Hz}$. Spectra between $0.5-20 \mathrm{~Hz}$ were investigated. The average spectral power was computed across all epochs of a given sleep stage (N2, N3, NREM, REM), separately for the entire night, the first half, and second half of the night. 
The power spectra were computed for the first and second halves of the sleep episodes, as well as for the entire night, separately for NREM sleep, stages N2 and N3, and REM sleep.

Current source density analysis

To investigate how GHB affected physiological EEG oscillations in NREM sleep, 10 min segments around the maximum of slow-wave activity (SWA) values in the first NREM sleep episode following $\mathrm{GHB} /$ placebo administration were extracted for further analyses. All used segments were located in the peak phase of drug action $\left(t_{45}-t_{75}\right)$ [30]. The procedure is depicted in Fig. 1, indicating the time point of GHB (red triangle) and placebo (black triangle) administration, and segment extraction for current source density (CSD) analysis (marked with a green arrow) in a representative individual. Extracted segments were preprocessed using Brain Vision Analyzer 2 software (Brain Products $\mathrm{GmbH}$ ). First, EEG data were re-referenced to the average of all scalp electrodes. Second, a bandpass filter from 0.5 to $40 \mathrm{~Hz}$ was applied to the EEG data, to attenuate channel drifts and satisfy the assumption of stationarity necessary for computing independent component analysis. Third, movement-related artifacts were visually identified and excluded. Fourth, the preprocessed segment was segmented into $12 \mathrm{~s}$ segments and exported for further analysis. The minimal segment number used for CSD analyses was 45 . Two subjects had to be excluded from CSD analysis because of insufficient data quality. The CSD was analyzed using exact low-resolution brain electromagnetic topography (eLORETA, http://www.uzh.ch/keyinst/ loreta.htm), a widely used mathematical approach to estimate the sources of electrical currents measured with scalp EEG. eLORETA computes a three-dimensional EEG CSD map by applying a three-spherical shell model restricting the solution space to the gray matter and hippocampus, resulting in 6239 different voxels of $5 \times 5 \times 5 \mathrm{~mm}$ each. The anatomical brain model used for the computation of intracerebral CSD values was registered on a digitized average magnetic resonance imaging (MRI) brain of the Talairach and Tournoux atlas (Brain Imaging Centre, Montreal Neurological Institute). To compute spectral density $\left(\mu \mathrm{A} / \mathrm{mm}^{2}\right)$, the signal was split into delta $(0.5-4.5 \mathrm{~Hz})$, theta $(4.5-8 \mathrm{~Hz})$, alpha $(8-12 \mathrm{~Hz})$, sigma $(12-16 \mathrm{~Hz})$, and beta $(16-20 \mathrm{~Hz})$ frequency bands.

\section{Lagged phase synchronization analysis}

The lagged-phase synchronization (LPS) analysis implemented in the eLORETA software was used to characterize differences in functional connectivity among brain areas in GHB-augmented sleep when compared with placebo. Phase synchronization of neuronal oscillations has been repeatedly shown to reflect the coordination of activity among distinct brain regions [31, 32]. Quantification of LPS thus offers a valuable tool to investigate physiological processes underlying functional connectivity between distinct neuronal assemblies [33].

This method has undergone cross-modal validation from both diffusion tensor imaging [34] and functional MRI [35]. LPS allows calculating the functional connectivity among regions of interest (ROI) for all defined frequency bands. Six brain regions, which were significantly affected by GHB (as revealed by the CSD analysis), were chosen for ROI analyses: anterior cingulate cortex (ACC; $X=1, Y=29, Z=14$ ), posterior cingulate cortex (PCC; $X=1$, $Y=-51, \quad Z=8$ ), left dorsolateral prefrontal cortex (IdIPFC; $X=-39, Y=40, Z=28$ ), right dorsolateral prefrontal cortex (rdIPFC; $X=39, Y=40, Z=28$ ), left parahippocampal gyrus (IPHG; $X=-27, Y=-40, Z=-14)$, and right parahippocampal gyrus (rPHG; $X=27, Y=-40, Z=-14$ ).

\section{Statistical analyses}

A linear mixed-effects model, with condition (GHB vs. placebo) as within-subject factor and subject ID as random effect was employed on R (RStudio Version 1.0.136; RStudio, Inc.) for the analyses of the sleep variables (R-package "Ime4," Version 1.1-15). For all applied models, normal $Q-Q$ plots were applied, demonstrating normality of the residuals. Moreover, the assumption of homoscedasticity and linearity was verified using a TukeyAnscombe (residuals vs. fitted) plot. Furthermore, autocorrelation plots did not reveal any autocorrelation among the residuals. $P$ values of post-hoc tests (R-package "emmeans," Version 1.2.1) were corrected for multiple comparison using Benjamini-Hochberg correction of the false discovery rate [36]. Spectral data were statistically analyzed with a linear mixed-effects model (R-package "nlme;" Version 3.1), while controlling for inter-frequency bin correlations, by an autoregressive moving average model and comparing bin-wise with general linear hypothesis tests. Statistical differences in CSD between the conditions were calculated using a nonparametric mapping approach as implemented in the eLORETA software [37]. A voxel-by-voxel-dependent $t$-test was used, whereby the contrast between the conditions for all frequency bands were calculated separately. Corrections for multiple comparisons were performed across voxels and frequency bands, applying a randomization strategy with 5000 permutations. Moreover, statistical regularization (variance smoothing parameter for $t$-statistic at $5 \%$ ) was applied.

Statistical differences in LPS between the conditions were calculated using a nonparametric mapping approach (as implemented in the eLORETA software) [37]. A paired $t$-test was used, calculating the contrasts between the conditions for all frequency bands. Corrections for multiple comparisons were performed across ROls and frequency bands, applying a randomization strategy with 5000 permutations.

\section{RESULTS}

Sleep-wake diary and actigraphy

The data reported in the sleep diaries during the 2 weeks before the first experimental night and the week between the two experimental blocks revealed mean bedtime at 23:34 $\pm 34 \mathrm{~min}$, mean wake-up time at 7:25 $\pm 27 \mathrm{~min}$, and a mean sleep duration of $472 \pm 21 \mathrm{~min}$. The analyses of the actigraphic recordings led to comparable results (Bedtime: 23:30 $\pm 27 \mathrm{~min}$; Wake-up time: $7: 30 \pm 20 \mathrm{~min}$; sleep duration $480 \pm 12 \mathrm{~min}$ ).

Sleep variables

GHB robustly prolonged N3 sleep and NREM sleep, and shortened REM sleep when compared with placebo (Fig. 2). Although these effects were significant when the entire night was considered (all $p<0.05)$, they were even more pronounced when the analysis was restricted to the second half of the night (all $p<0.001$; Supplementary Table S1). No differences $(p>0.05)$ between GHB and placebo were observed for sleep efficiency, the durations of TST, duration of N1 and N2 sleep, and wakefulness.

\section{EEG power spectra}

Sleep architecture and the evolution of EEG power between 0.5 and $30 \mathrm{~Hz}$, as well as EEG SWA (power in the $0.75-4.5 \mathrm{~Hz}$ range) in a representative individual in the placebo and GHB conditions are plotted in Fig. 1. Reflecting the declining trend in sleep intensity across the sleep episode, SWA in the second half of the night is drastically reduced compared with the first half of the night in the placebo condition. Although the volunteer returned to sleep almost immediately following both GHB and placebo administration, a prominent GHB-induced increase in SWA in NREM sleep episodes 3 (at around 03:00) and 4 (at around 05:00) is clearly apparent. On the group level, the compound increased EEG activity in virtually all bins of delta/theta $(0.5-8 \mathrm{~Hz})$ frequencies in stages N2 and N3, as well as when these stages were analyzed together (Fig. 3). In N2 sleep and combined stages N2 and N3 (NREM sleep), GHB also reduced power in the frequency range of sleep spindles (13-15 Hz). In REM sleep, the differences between 

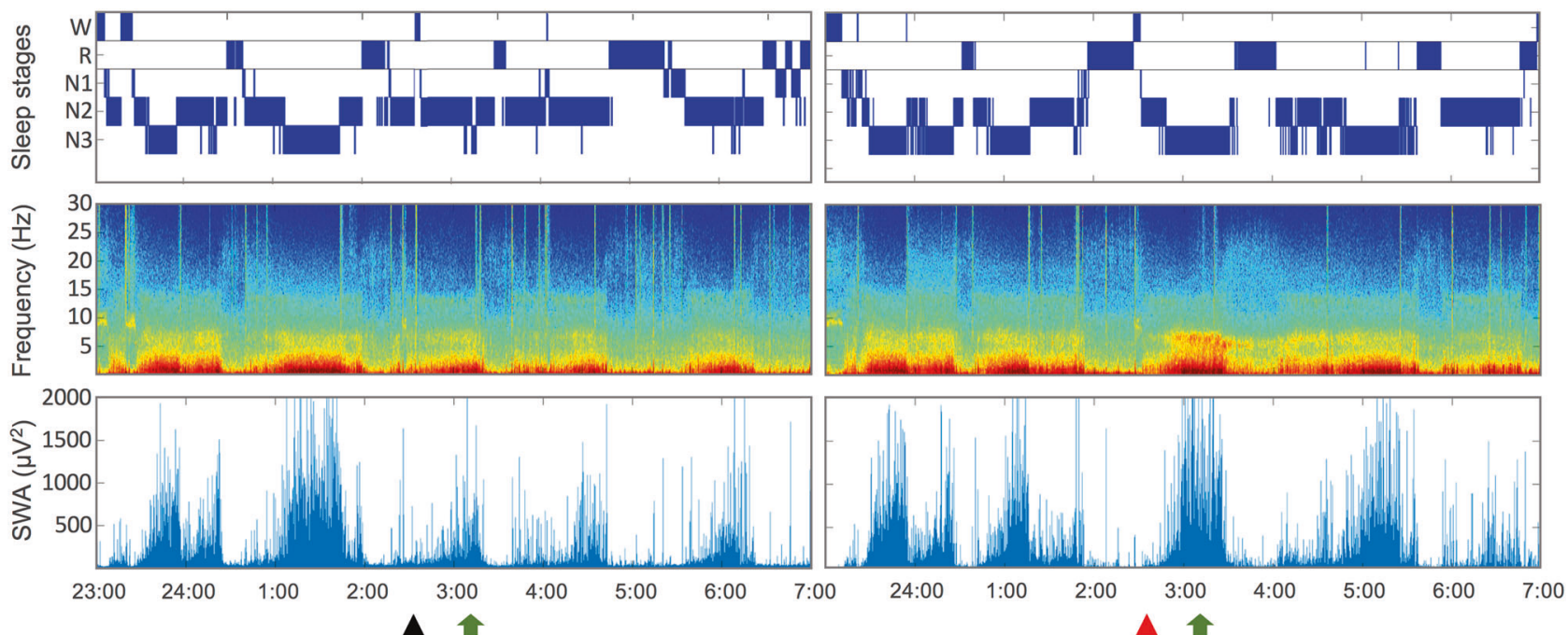

clock time $(\mathrm{h})$

Fig. 1 Hypnogram (top row), time frequency analysis (middle row), and course of slow-wave activity (bottom row; SWA, power density within $0.75-4.5 \mathrm{~Hz} ; \mathrm{C} 3 \mathrm{~A} 2$ derivation) of a single subject $(N=1)$ after intake of a placebo (left panel) and $\mathrm{GHB}$ (right panel). The triangles indicate the time point of placebo (black) GHB (red) administration. Arrows indicate time point of EEG segment extraction for eLORETA analysis (CSD and LPS). N1 stage 1 sleep; N2 stage 2 sleep, N3 stage 3 sleep; R, REMS; W, wake
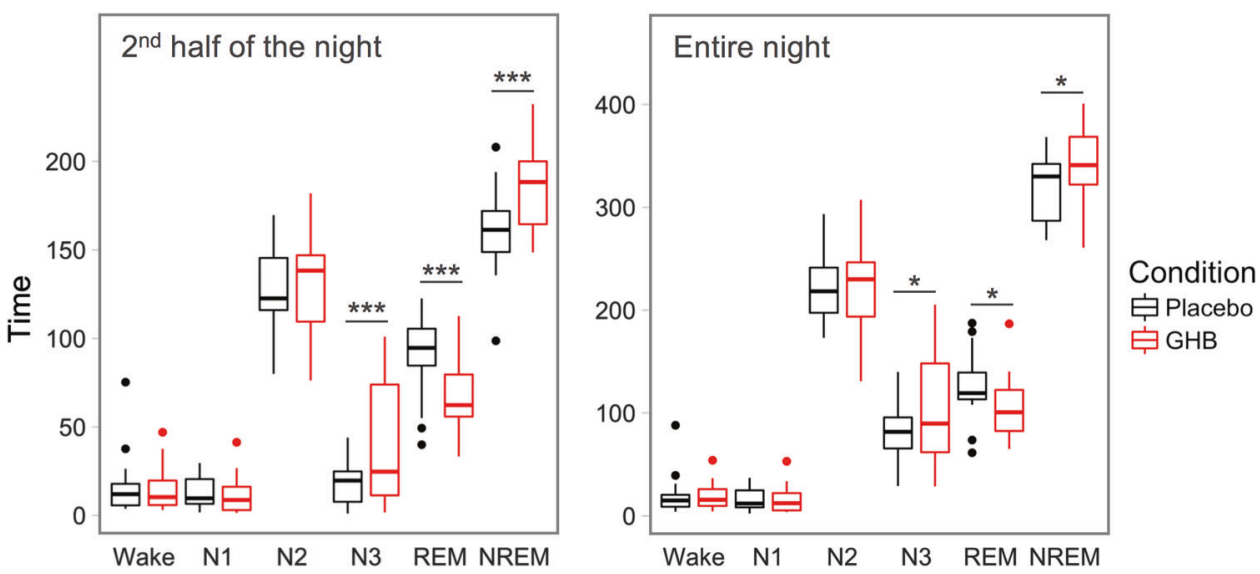

Fig. 2 Duration of sleep stages in black (placebo) and GHB (red) conditions. Boxplots represent visually scored wakefulness and the sleep stages N1-N3 and REM sleep. Horizontal lines indicate median values; boxes extend from the 25th to the 75th percentile; vertical extending lines denote adjacent values (i.e., the most extreme values within 1.5 interquartile range of the 25th and 75 th percentile); dots denote observations outside the range of adjacent values. NREM = combined stages N2 and N3 [29]. Entire night: from 23:00 to 07:00. Second half of the night: from 02:30 (time of GHB and placebo intake) to 07:00. Asterisks indicate significant differences between GHB and placebo conditions: ${ }^{*} p<0.05 ;{ }^{* * *} p<0.001$ (Benjamini-Hochberg corrected post hoc $t$-tests, $\left.n=20\right)[9,27,69-74]$

GHB and placebo were restricted to the $<1 \mathrm{~Hz}$ and $6-8 \mathrm{~Hz}$ bands. The GHB-induced changes in sleep EEG power spectra reflect more intense sleep when compared with placebo.

\section{Current source density}

CSD analysis in the first NREM sleep episode following GHB and placebo intake was performed for the EEG delta, theta, alpha, sigma, and beta ranges (see Fig. 1 for an illustration of time point when the CSD analyses were conducted). These analyses revealed increased CSD in delta $(0.5-4.5 \mathrm{~Hz})$ and theta $(4.5-8 \mathrm{~Hz})$ ranges following the administration of GHB when compared with placebo. The increase was distributed approximately symmetrically in both hemispheres. In the delta range, the following brain areas were affected by the drug: parahippocampal gyrus, lingual gyrus, fusiform gyrus, PCC, ACC, cuneus, medial frontal gyrus, subcallosal gyrus, rectal gyrus, inferior frontal gyrus, orbital gyrus, superior frontal gyrus, and middle frontal gyrus (Fig. 4, upper panel). In the theta range, the following brain areas were affected by the drug: medial frontal gyrus, ACC, superior frontal gyrus, orbital gyrus, rectal gyrus, middle frontal gyrus, inferior frontal gyrus, subcallosal gyrus, insula, superior temporal hyrus, middle temporal gyrus, uncus, parahippocampal gyrus, and inferior temporal gyrus (Fig. 4, lower panel). Supplementary Table S2 (Supplementary Material) summarizes the results of the statistical analyses, reporting significantly affected brain regions, the number of significant voxels within the brain regions, Talairach coordinates of the maximal $t$-value, and the corresponding $t$ - and $p$-values.

\section{Lagged-phase synchronization}

As illustrated in Fig. 5, functional connectivity (or synchronization) in the theta range $(4.5-8 \mathrm{~Hz})$ was significantly increased after GHB 

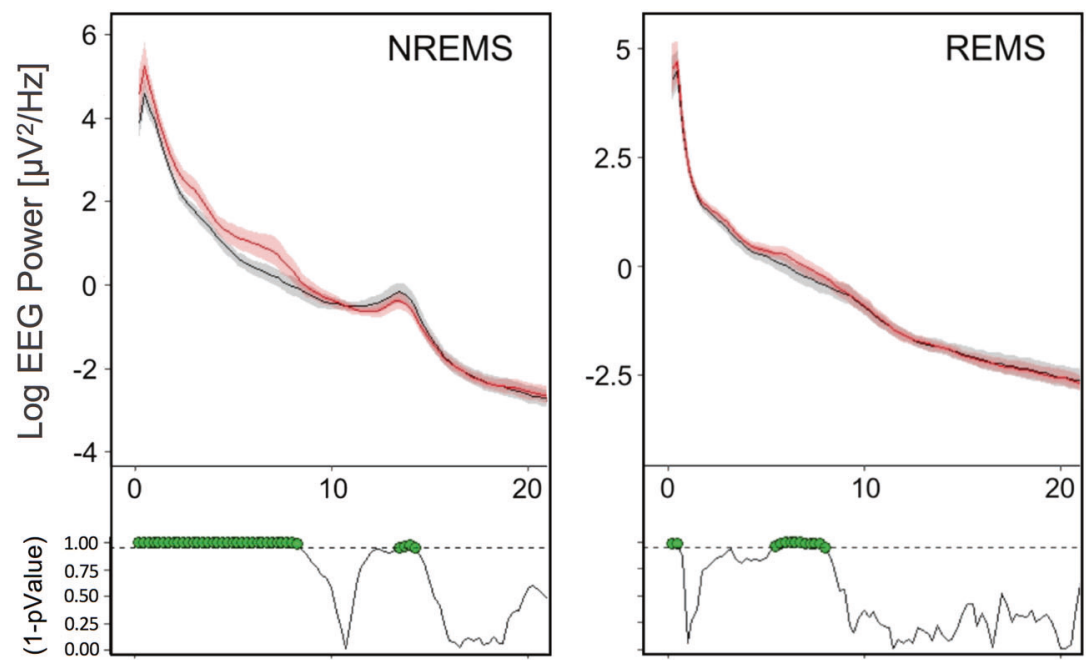

Condition
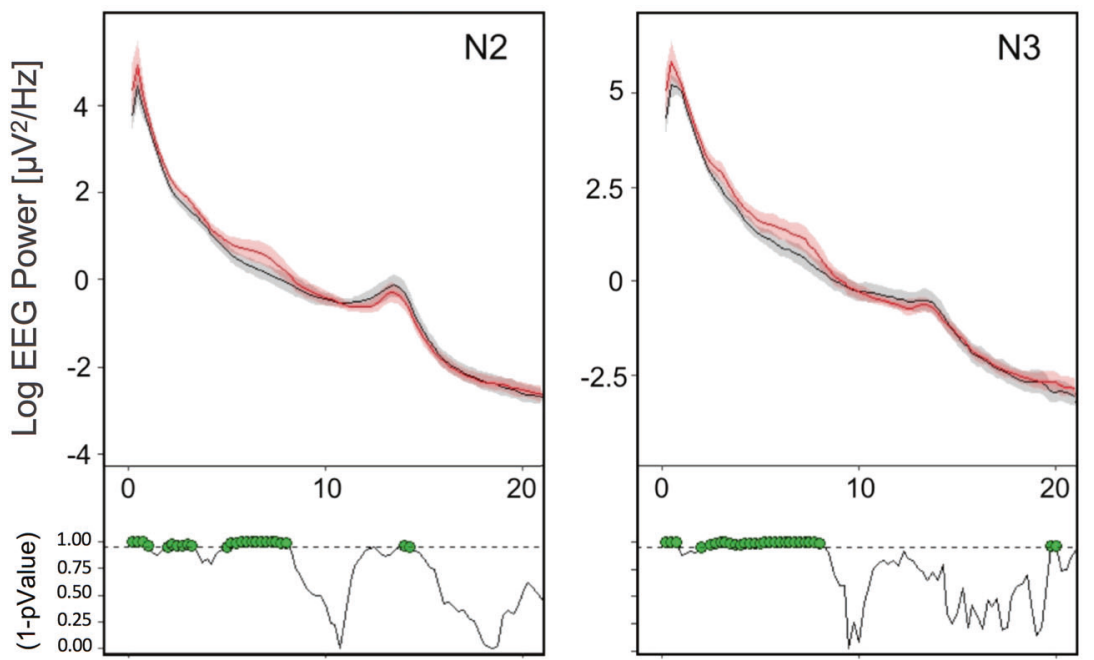

Frequency $[\mathrm{Hz}]$

Fig. 3 Absolute EEG power spectra (C3-A2 derivation) in the second half of the night (02:30-07:00) following GHB (red lines) and placebo (black lines) administration. Mean values and confidence intervals (shading) were plotted for NREM sleep (combined stages N2 and N3), REM sleep, and stages N2 and N3 separately. Green dots at the bottom of the panels indicate frequency bins $(0.25 \mathrm{~Hz}$ resolution), which differed significantly from placebo ( $p<0.05$, linear mixed-effects model with condition as within-subject factor)

when compared with placebo between ACC and PCC, ACC and IPHG, ACC and rdIPFC, ACC and IdIPFC, IdIPFC and PCC, and between rdIPFC and rPHG. Supplementary Table S3 (Supplementary Material) summarizes the $t$ - and $p$-values of the corresponding connections. No significant effects of GHB on functional connectivity were found in the delta, alpha, sigma, or beta ranges (results not shown).

\section{DISCUSSION}

Our study provides evidence that a therapeutic dose of GHB induces a biomimetic enhancement of sleep intensity when administered to healthy adults under low homeostatic sleep pressure. More specifically, $50 \mathrm{mg} / \mathrm{kg} \mathrm{GHB}$ given at 2:30 a.m., at the time of typical dosing of GHB dose in narcolepsy treatment, increased the duration of deep NREM sleep (N3) at the cost of REM sleep. Furthermore, it enhanced EEG delta and theta activity in NREM and REM sleep, while reducing spindle frequency activity in more superficial N2 sleep. Source localization suggested that GHB augmented delta band activity in NREM sleep especially in the
ACC, in the medio-prefrontal cortex, in both PHG and in the PCC. In the theta band, the increase predominated in the prefrontal cortex, ACC, and both temporal poles. The functional connectivity in this frequency range during GHB-augmented sleep was strengthened among bilateral dIPFC, ACC, PCC, and bilateral PHG. Collectively, the GHB-induced changes in sleep and the sleep EEG resemble the characteristics of recovery sleep after sleep deprivation.

Clinically, GHB is the first-line treatment of excessive daytime sleepiness and cataplexy in narcolepsy type- 1 and has recently been shown to be an effective novel treatment option for sleep-wake disturbances in Parkinson's disease $[10,38]$. Although it has been suggested that GHB may augment the regenerative functions of sleep $[9,39]$ and activate similar physiological mechanisms as sleep deprivation [18], the neurophysiological signature of GHB-augmented sleep has not yet been systematically investigated. In fact, the two available studies that examined the effects of GHB on the sleep EEG in healthy volunteers in conditions of either attenuated or enhanced physiological sleep came to different conclusions. Although one 


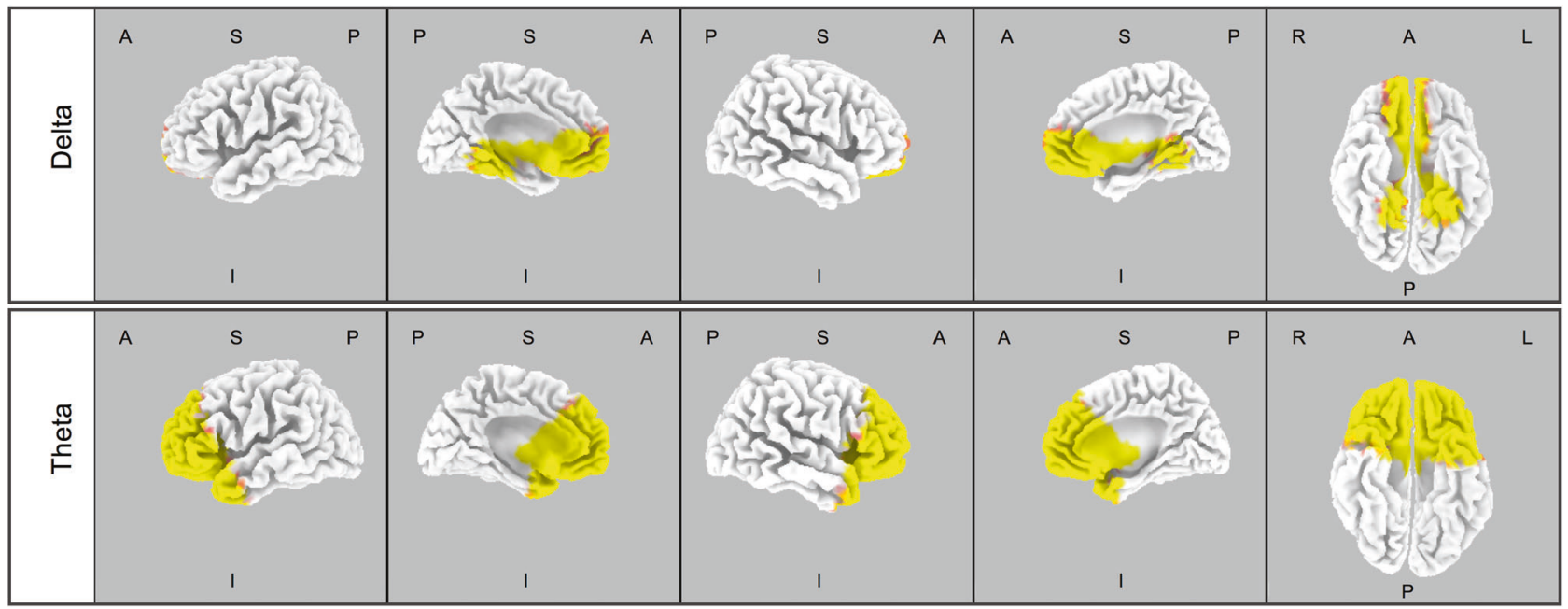

Fig. 4 Statistical maps depicting the brain regions which differ between GHB and placebo in delta $(0.5-4.5 \mathrm{~Hz}$; upper panel) and theta (4.5-8 Hz Hz; lower panel) frequencies in the first NREM sleep episode following GHB and placebo administration ( $n=18)$. Significant log $\mathrm{F}$ ratios at $p<0.05$ are illustrated in yellow (GHB $>$ placebo) and log $\mathrm{F}$ ratios at $p<0.1$ are depicted in red (GHB $>$ placebo). Rows from top to down: delta $(0.5-4.5 \mathrm{~Hz})$ and theta $(4.5-8 \mathrm{~Hz})$. A, anterior; I, inferior; L, left; P, posterior; R, right; S, superior

noted that GHB-induced slow waves are functionally dissimilar to physiological slow waves [15], the other indicated that enhancing slow-wave sleep with GHB reduces the homeostatic response to sleep loss and thus promotes physiological sleep-wake mechanisms [40]. Although in our study no experimental manipulation of homeostatic sleep propensity was performed, the here shown findings support the latter hypothesis.

The prevalence of slow-wave sleep and EEG delta/theta frequencies in NREM sleep decrease in the course of a sleep episode (see Fig. 1) are reduced after a daytime nap and predictably enhanced after extended wakefulness [41]. It is widely accepted that recovery sleep following prolonged wakefulness is physiologically intensified when compared with baseline sleep. Here, at a time of reduced sleep pressure in the second half of a sleep episode, we observed striking similarities between the effects of GHB and those induced by sleep deprivation. Following GHB intake, the duration of deep NREM sleep (stage N3) was increased, whereas REM sleep duration was reduced. Moreover, GHB increased delta and theta activity, and attenuated spindle frequency activity in NREM sleep, and also enhanced theta power in REM sleep. Very similar changes are well established after sleep deprivation $[20,22,23,42]$. The CSD analysis further corroborated the similarity with the physiological effect and revealed striking topographical overlaps between GHB-modulated and recovery sleep, including increased delta and theta power over frontal recording sites in NREM sleep $[22,43,44]$.

On a molecular level, most of GHB's sleep electrophysiological effects can most likely be attributed to low-affinity agonistic binding to $G A B A_{B}$ receptors [45], and work in mice, rats, and cats suggests that $G A B A_{B}$ receptors contribute to endogenous sleep oscillations [46-48]. Given the widespread expression of $G_{A B A_{B}}$ receptors throughout the brain [49], the area-specific increase in delta/theta power may appear astonishing. Nevertheless, GHB may predominantly increase low-frequency power in NREM sleep in brain areas, which are also increased under conditions of elevated homeostatic sleep pressure $[22,43]$. On the other hand, although the present study is consistent with experimental and theoretical evidence that agonism at $\mathrm{GABA}_{B}$ receptors increases deep sleep and EEG delta power $[40,48]$, it cannot be excluded that also other neurotransmitter systems contribute to the promotion of EEG slow waves by GHB. For example, both GHB and $G_{A B A}$ receptors affect the serotonergic system [50], which also contributes to changes in the EEG spectrum, which are similar to those seen after sleep deprivation [51].

Apart from frontal-central sites, GHB enhanced delta activity in the PHG, the fusiform gyrus, and the PCC. The functional connectivity analysis further revealed strengthened LPS among these areas after drug intake. A similar effect was found in a previous study, when investigating the effects of GHB on waking resting state LPS in healthy subjects [52]. Nevertheless, in waking subjects GHB's effects on CSD distinctly differ from those found in this study, indicating that GHB's neurophysiological effects may depend on the subject's state of consciousness.

In vitro studies demonstrated that bath application of GHB to thalamo-cortical neurons hyperpolarized these cells into a voltage range, -65 to $-75 \mathrm{mV}$, at which the rhythmic pacemaker oscillations of the cell membrane exhibits a frequency of 0.5 to $4.0 \mathrm{~Hz}[53,54]$. A similar hyperpolarization was also observed in hippocampal neurons, which might give a mechanistic explanation for the increased delta power in the PHG [55].

Intriguingly, these structures not only share tight anatomical connections but are also functionally associated in the process of learning, memory encoding, and retrieval $[56,57]$. Consequently, damages within these structures-as found in Alzheimer's disease - severely affect learning and memory [58, 59]. Likewise prefrontal atrophy in elderly and Alzheimer's patients was associated with hippocampal-dependent memory impairment and disrupted NREMS [60]. Thus, GHB's ability to induce slow oscillations and strengthen functional connectivity in those brain sites, might underline its clinical potential as regenerative sleepaid in neurodegenerative disorders (for review, see refs. [38, 39]).

GHB not only affected NREM sleep, but also had a significant impact on REM sleep. In accordance with previous research $[15,40]$, GHB acutely reduced REM sleep and increased EEG delta and theta activity also in this sleep state. Despite its nominal reduction, more than $1 \mathrm{~h}$ of REM sleep persisted during the peak phase of pharmacological action, indicating that REM sleep can prevail under elevated $\mathrm{GABA}_{\mathrm{B}}$-ergic tone, which may favor EEGdefined deep NREM sleep. This finding may reflect the well-known sleep-dependent disinhibition of and high endogenous pressure for REM sleep in the early morning hours, at the time of GHB administration $[20,61,62]$. Nevertheless, even in this condition of high endogenous REM sleep pressure, we found no support for the notion that the stimulation of $G_{A B A}$ receptors favors 


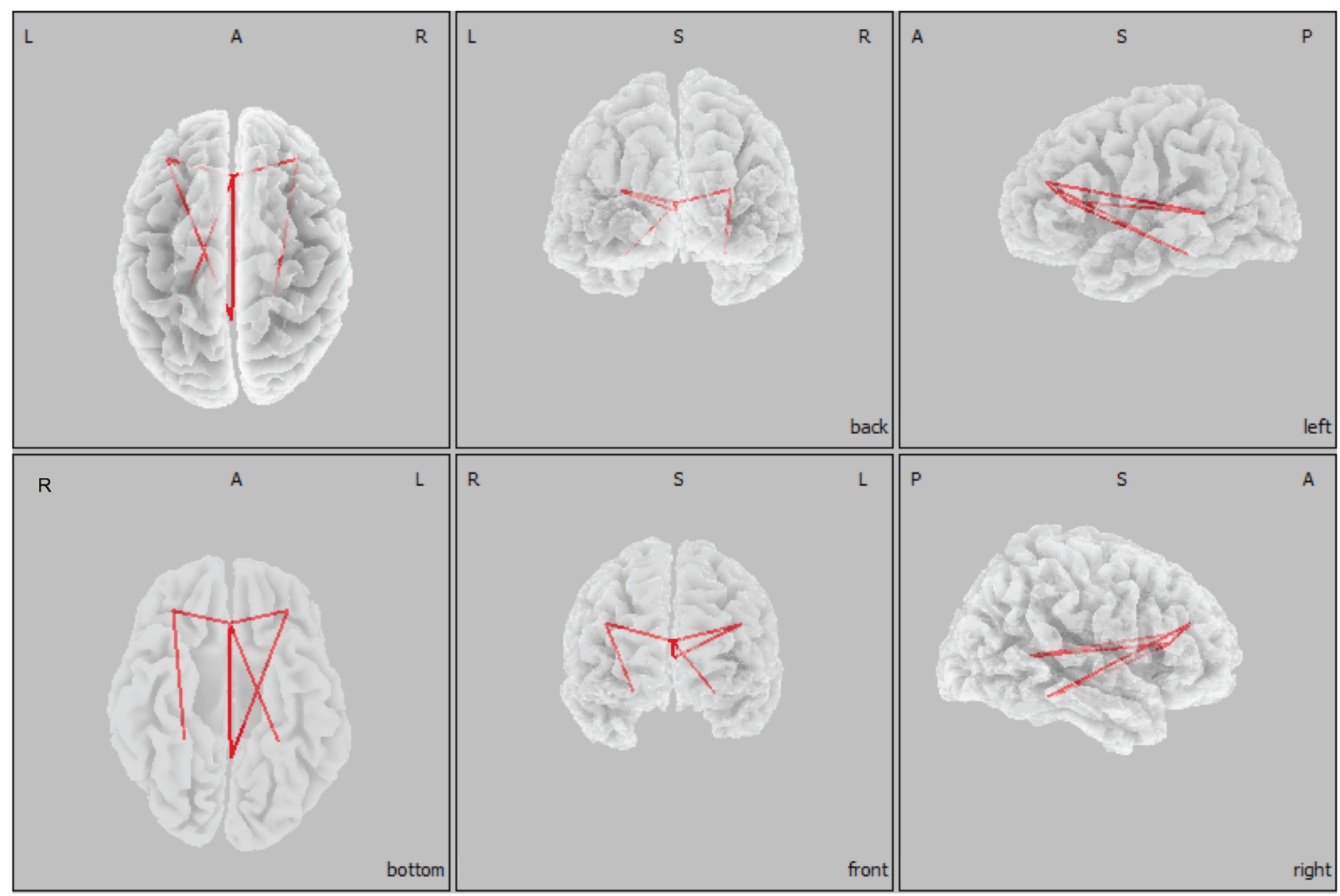

Fig. 5 Region of interest (ROI)-based analysis of LPS in the first NREM sleep episode following GHB and placebo administration ( $n=18$ ). Based on the results of the CSD analysis, a network of six cortical brain regions was significantly modulated by $\mathrm{GHB}$ in the theta range $(4.5-8 \mathrm{~Hz})$. This network included the anterior and posterior cingulate gyri, the left and right dorsolateral prefrontal cortices, and the left and right parahippocampal gyri, which were defined as ROIs. The red lines indicate significantly increased LPS between ROIs after GHB when compared with placebo. A, anterior; I, inferior; L, left; P, posterior; R, right; S, superior

sleep-onset REM sleep episodes that was suggested by previous work [15]. It is likely to be that in the present study, REM sleep was reduced as a result of increased N3 sleep duration, yet an ad libitum sleep opportunity would be required to study the acute and subacute effects of GHB on REM sleep expression.

Similar to the spectral changes in NREM sleep, the increased delta/theta power in REM sleep is reminiscent of the impact of prolonged waking on recovery sleep [43, 44,63]. These changes may underlie GHB's ability to restore REM sleep functions in neuropsychiatric disorders, including narcolepsy and major depressive disorder [9]. On the other hand, GHB was reported to reduce arousals during REM sleep episodes in healthy volunteers [19]. Future studies should investigate the interaction between GHB and sleep deprivation, to more conclusively tackle the question whether the same mechanisms are activated during GHB-augmented sleep and recovery sleep after prolonged wakefulness. Although comparisons between pharmacologically and physiologically induced alterations of sleep mechanisms must be made with caution, several studies indicate that GHB-augmented sleep not only shows neurophysiological overlaps but also functional similarities with physiological sleep. For example, acute GHB administration spared energy metabolism and, similar to well several wake-promoting neurotransmitter systems [50, 64-68], induced growth hormone release in a similar fashion as natural slow-wave sleep [14], and reduced the consequences of sleep loss on measures of alertness and attention [40]. Intriguingly, even though all participants in the present study were young, healthy, and well rested ( $8 \mathrm{~h}$ sleep), nocturnal GHB promoted morning vigilance as quantified on the psychomotor vigilance task $3 \mathrm{~h}$ after awakening (median reaction time: $297.9 \pm 26.04 \mathrm{~ms}$ vs. $290.25 \pm 25.87 \mathrm{~ms} ; n=20, p<0.05$, Benjamini-Hochberg corrected post-hoc test). Moreover, no detrimental effects on subjective sleepiness and mood were found upon awakening, indicating the absence of pharmacological hangover effects. Taken together, these convergent findings support the view that GHB enhances restorative aspects of sleep rather than just inducing a sedative state. Interestingly, GHB induced qualitatively uniform effects on several neurophysiological parameters across all subjects. Nevertheless, at the same time, high inter-individual differences in the strength of the induced effects were observed. We speculate that both pharmacokinetic and pharmacodynamics differences across subjects may account for this variance, including individual differences in drug metabolism, $G A B A_{B}$ receptor density, and distribution pattern of $\mathrm{GABA}_{B}$ receptor isoforms [65]. Further studies are necessary to elucidate the mechanisms underlying the high inter-individual variations.

\section{Limitations}

When interpreting the current study, some limitations should be kept in mind. All study subjects where young healthy men, which limits the generalizability of the results. Moreover, the intervention comprised one single administration, whereas in a clinical setting, chronic administration would be the rule. Thus, based on our findings, long-term GHB effects on sleep neurophysiology are difficult to predict. As the present study did not include a sleep deprivation intervention, further studies employing both sleep deprivation and GHB administration are needed to support the 
suggestion of biomimetic sleep enhancement by GHB. Finally, despite LORETA's accuracy in localizing cortical electrical sources, the algorithm is not able to detect subcortical sources. In other words, neural structures that are crucially involved in sleep-wake regulation, including the thalamus, hypothalamus and basal ganglia, cannot be investigated with this method. Thus, it remains unclear whether and how these brain areas contribute to GHB's effects on sleep. Moreover, the anatomical brain model used for the computation of intracerebral CSD values was registered on a digitized average MRI brain of the Talairach and Tournoux atlas. Using individual MRI T1-brain images may have yielded more accurate source estimations.

\section{CONCLUSION}

In the present study, a detailed sleep neurophysiological signature of GHB-augmented sleep under low homeostatic sleep pressure was established. Intriguingly, the GHB-induced changes in sleep architecture, sleep EEG power spectra, CSD, and functional connectivity mimicked many characteristics of physiologically intensified sleep, such as in recovery sleep following sleep deprivation. Thus, GHB's potential to promote physiological sleep mechanisms and its clinical application to consolidate sleep and improve waking quality in neurological and neuropsychiatric disorders should be further investigated.

\section{FUNDING AND DISCLOSURE}

The study was supported by grants from the Swiss National Science Foundation (SNSF) to HPL (grant number 320030_163439) and the Clinical Research Priority Program Sleep \& Health of the University of Zurich. DD had full access to all data of the study and takes responsibility for their integrity and the accuracy of the data analyses. The authors declare no competing interests.

\section{ACKNOWLEDGEMENTS}

We thank Vinnie Kandra for her dedicated assistance in data collection and participant recruitment. Moreover, we thank Antonio Fernandez Guerrero for his valuable comments on the CSD and LPS analyses.

\section{ADDITIONAL INFORMATION}

Supplementary Information accompanies this paper at (https://doi.org/10.1038/ s41386-019-0382-z).

Publisher's note: Springer Nature remains neutral with regard to jurisdictional claims in published maps and institutional affiliations.

\section{REFERENCES}

1. Fernandez-Mendoza J, Vgontzas AN. Insomnia and its impact on physical and mental health. Curr Psychiatr Rep. 2013. https://doi.org/10.1007/s11920-0130418-8

2. Ohayon MM, Caulet M, Lemoine P. Comorbidity of mental and insomnia disorders in the general population. Compr Psychiatry. 1998. https://doi.org/ 10.1016/S0010-440X(98)90059-1

3. Weissman MM, Greenwald S, Niño-Murcia G, Dement WC. The morbidity of insomnia uncomplicated by psychiatric disorders. Gen Hosp Psychiatry. 1997. https://doi.org/10.1016/S0163-8343(97)00056-X

4. Wafford KA, Ebert B. Emerging anti-insomnia drugs: tackling sleeplessness and the quality of wake time. Nat Rev Drug Discov. 2008. https://doi.org/10.1038/ nrd2464

5. Winsky-Sommerer R. Role of GABAA receptors in the physiology and pharmacology of sleep. Eur J Neurosci. 2009. https://doi.org/10.1111/j.14609568.2009.06716.x

6. Akeju O, Brown EN. Neural oscillations demonstrate that general anesthesia and sedative states are neurophysiologically distinct from sleep. Curr Opin Neurobiol. 2017. https://doi.org/10.1016/j.conb.2017.04.011
7. Landolt HP, Gillin JC. GABA(A1a) receptors: Involvement in sleep regulation and potential of selective agonists in the treatment of insomnia. CNS Drugs. 2000. https://doi.org/10.2165/00023210-200013030-00005

8. Feinberg I, Maloney T, Campbell IG. Effects of hypnotics on the sleep EEG of healthy young adults: new data and psychopharmacologic implications. J Psychiatr Res. 2000. https://doi.org/10.1016/S0022-3956(00)00038-8

9. Mamelak M. Narcolepsy and depression and the neurobiology of gammahydroxybutyrate. Prog Neurobiol. 2009. https://doi.org/10.1016/j.pneurobio.2009.07.004

10. Büchele F, Hackius M, Schreglmann SR, Omlor W, Werth E, Maric A, et al. Sodium oxybate for excessive daytime sleepiness and sleep disturbance in Parkinson disease. JAMA Neurol. 2018. https://doi.org/10.1001/jamaneurol.2017.3171

11. Huang Y-S, Guilleminault C. Narcolepsy: action of two gamma-aminobutyric acid type B agonists, baclofen and sodium oxybate. Pediatr Neurol. 2009;41:9-16. https://doi.org/10.1016/j.pediatrneurol.2009.02.008

12. Ondo WG, Perkins T, Swick T, Hull KL, Jimenez JE, Garris TS, et al. Sodium oxybate for excessive daytime sleepiness in Parkinson disease. Arch Neurol. 2008;65:1337-1340. https://doi.org/10.1001/archneur.65.10.1337

13. Swick TJ. Sodium oxybate: a potential new pharmacological option for the treatment of fibromyalgia syndrome. Ther Adv Musculoskelet Dis. 2011;3:167-178. https://doi.org/10.1177/1759720X11411599

14. Van Cauter E, Plat L, Scharf MB, Leproult R, Cespedes S, L'Hermite-Balériaux M, et al. Simultaneous stimulation of slow-wave sleep and growth hormone secretion by gamma-hydroxybutyrate in normal young men. J Clin Investig. 1997;100:745-753. https://doi.org/10.1172/JCl119587

15. Vienne J, Lecciso G, Constantinescu I, Schwartz S, Franken P, Heinzer R, et al. Differential effects of sodium oxybate and baclofen on EEG, sleep, neurobehavioral performance, and memory. Sleep. 2012;35:1071-83. https://doi.org/ 10.5665/sleep.1992

16. Tononi G, Cirelli C. Sleep and the price of plasticity: from synaptic and cellular homeostasis to memory consolidation and integration. Neuron. 2014. https://doi. org/10.1016/j.neuron.2013.12.025

17. Walker MP. The role of sleep in cognition and emotion. Ann N Y Acad Sci. 2009. https://doi.org/10.1111/j.1749-6632.2009.04416.x

18. Morawska MM, Buchele F, Moreira CG, Imbach LL, Noain D, Baumann CR. Sleep modulation alleviates axonal damage and cognitive decline after rodent traumatic brain injury. J Neurosci. 2016;36:3422-3429. https://doi.org/10.1523/ JNEUROSCI.3274-15.2016

19. Lapierre O, Montplaisir J, Lamarre M, Bedard MA. The effect of gammahydroxybutyrate on nocturnal and diurnal sleep of normal subjects: further considerations on REM sleep-triggering mechanisms. Sleep. 1990. https://doi.org/ 10.1093/sleep/13.1.24

20. Borbely AA. A two process model of sleep regulation. Hum Neurobiol. 1982. https://doi.org/10.1111/jsr.12371

21. Borbely AA, Achermann P. Sleep homeostasis and models of sleep regulation [see comments]. J Biol Rhythms. 1999. https://doi.org/10.1016/B978-1-4160-66453.00037-2

22. Finelli LA, Borbély AA, Achermann P. Functional topography of the human nonREM sleep electroencephalogram. Eur J Neurosci. 2001. https://doi.org/ 10.1046/j.0953-816X.2001.01597.x

23. Bersagliere A, Pascual-Marqui RD, Tarokh L, Achermann P. Mapping slow waves by EEG topography and source localization: effects of sleep deprivation. Brain Topogr. 2017. https://doi.org/10.1007/s10548-017-0595-6

24. Holst SC, Bersagliere A, Bachmann V, Berger W, Achermann P, Landolt H-P. Dopaminergic role in regulating neurophysiological markers of sleep homeostasis in humans. J Neurosci. 2014. https://doi.org/10.1523/JNEUROSCI.412813.2014

25. Bachmann V, Klein $C$, Bodenmann S, Schäfer N, Berger $W$, Brugger $P$, et al. The BDNF Val66Met polymorphism modulates sleep intensity: EEG frequency- and state-specificity. Sleep. 2012. https://doi.org/10.5665/sleep.1690

26. Jasper $\mathrm{HH}$. The ten-tweny electrode system of the international federation. Electroencephalogr Clin Neurophysiol. 1958. https://doi.org/10.1016/0013-4694 (58)90053-1

27. Retey JV, Adam M, Gottselig JM, Khatami R, Durr R, Achermann P, et al. Adenosinergic mechanisms contribute to individual differences in sleep deprivationinduced changes in neurobehavioral function and brain rhythmic activity. J Neurosci. 2006. https://doi.org/10.1523/JNEUROSCI.1538-06.2006

28. Valomon A, Holst SC, Borrello A, Weigend S, Müller T, Berger W, et al. Effects of COMT genotype and tolcapone on lapses of sustained attention after sleep deprivation in healthy young men. Neuropsychopharmacology. 2018. https://doi. org/10.1038/s41386-018-0018-8

29. Iber C, Ancoli-Israel S, Chesson AL Jr., Quan SF. The AASM manual for the scoring of sleep and associated events: rules, terminology and technical specifications. In: AASM manual for scoring sleep. 2007. https://doi.org/10.1002/ ejoc.201200111 
30. Liechti ME, Quednow BB, Liakoni E, Dornbierer D, Von Rotz R, Gachet MS, et al. Pharmacokinetics and pharmacodynamics of $\gamma$-hydroxybutyrate in healthy subjects. Br J Clin Pharmacol. 2016;81:980-988. https://doi.org/10.1111/bcp.12863

31. Buzsáki G, Draguhn A. Neuronal olscillations in cortical networks. Science. 2004. https://doi.org/10.1126/science.1099745

32. Uhlhaas PJ, Singer,W. Abnormal neural oscillations and synchrony in schizophrenia. Nat Rev Neurosci. 2010. https://doi.org/10.1038/nrn2774

33. Varela F, Lachaux JP, Rodriguez E, Martinerie J. The brainweb: Phase synchronization and large-scale integration. Nat Rev Neurosci. 2001. https://doi.org/ $10.1038 / 35067550$

34. Babiloni C, Lizio R, Carducci F, Vecchio F, Redolfi A, Marino S, et al. Resting state cortical electroencephalographic rhythms and white matter vascular lesions in subjects with alzheimer's disease: an italian multicenter study. J Alzheimer's Dis. 2011. https://doi.org/10.3233/JAD-2011-101710

35. De Ridder D, Vanneste S, Kovacs S, Sunaert S, Dom G. Transient alcohol craving suppression by rTMS of dorsal anterior cingulate: an fMRI and LORETA EEG study. Neurosci Lett. 2011. https://doi.org/10.1016/j.neulet.2011.03.074

36. Hochberg $Y$, Benjamini Y. More powerful procedures for multiple significance testing. Stat Med. 1990. https://doi.org/10.1002/sim.4780090710

37. Pascual-Marqui RD, Lehmann D, Koukkou M, Kochi K, Anderer P, Saletu B, et al. Assessing interactions in the brain with exact low-resolution electromagnetic tomography. Phil Transact R Soc A Math Phys Eng Sci. 2011. https://doi.org/ 10.1098/rsta.2011.0081

38. Mamelak M. Parkinson's disease, the dopaminergic neuron and gammahydroxybutyrate. Neurol Ther. 2018. https://doi.org/10.1007/s40120-018-0091-2

39. Mamelak M. Alzheimer's disease, oxidative stress and gammahydroxybutyrate Neurobiol Aging. 2007;28:1340-60. https://doi.org/10.1016/j.neurobiolaging. 2006.06.008

40. Walsh JK, Hall-Porter JM, Griffin KS, Dodson ER, Forst EH, Curry DT, et al. Enhancing slow wave sleep with sodium oxybate reduces the behavioral and physiological impact of sleep loss. Sleep. 2010;33:1217-25. http://www.ncbi.nlm. nih.gov/pubmed/20857869

41. Ong JL, Lo JC, Gooley JJ, Chee MWL. EEG changes accompanying successive cycles of sleep restriction with and without naps in adolescents. Sleep. 2017 https://doi.org/10.1093/sleep/zsx030

42. Maric A, Lustenberger C, Werth E, Baumann CR, Poryazova R, Huber R. Intraindividual increase of homeostatic sleep pressure across acute and chronic sleep loss: a high-density EEG study. Sleep. 2017. https://doi.org/10.1093/sleep/zsx122

43. Marzano C, Ferrara M, Curcio G, De Gennaro L. The effects of sleep deprivation in humans: topographical electroencephalogram changes in non-rapid eye movement (NREM) sleep versus REM sleep. J Sleep Res. 2010. https://doi.org/10.1111/ j.1365-2869.2009.00776.x

44. Borbély AA, Baumann F, Brandeis D, Strauch I, Lehmann D. Sleep deprivation: Effect on sleep stages and EEG power density in man. Electroencephalogr Clin Neurophysiol. 1981. https://doi.org/10.1016/0013-4694(81)90225-X

45. Kaupmann K, Cryan JF, Wellendorph P, Mombereau C, Sansig G, Klebs K, et al. Specific gamma-hydroxybutyrate-binding sites but loss of pharmacological effects of gamma-hydroxybutyrate in $\mathrm{GABA}(\mathrm{B})(1)$-deficient mice. Eur J Neurosci. 2003. https://doi.org/3013 [pii]

46. Gauthier P, Arnaud C, Gandolfo G, Gottesmann C. Influence of a GABA(B) receptor antagonist on the sleep-waking cycle in the rat. Brain Res. 1997. https://doi.org/ 10.1016/S0006-8993(97)00643-4

47. Juhász G, Emri Z, Kékesi KA, Salfay $O$, Crunelli V. Blockade of thalamic GABABreceptors decreases EEG synchronization. Neurosci Lett. 1994. https://doi.org/ 10.1016/0304-3940(94)90685-8

48. Vienne J, Bettler B, Franken P, Tafti M. Differential effects of GABAB receptor subtypes, \{gamma\}-hydroxybutyric acid, and Baclofen on EEG activity and sleep regulation. J Neurosci. 2010. https://doi.org/10.1523/JNEUROSCI.3145-10.2010

49. Bowery NGG, Hudson ALL, Price GWW. GABAA and GABAB receptor site distribution in the rat central nervous system. Neuroscience. 1987. https://doi.org/ 10.1016/0306-4522(87)90098-4

50. Szabo ST, Gold MS, Goldberger BA, Blier P. Effects of sustained gammahydroxybutyrate treatments on spontaneous and evoked firing activity of locus coeruleus norepinephrine neurons. Biol Psychiatr. 2004. https://doi.org/10.1016/j. biopsych.2003.12.013

51. Landolt HP, Meier V, Burgess HJ, Finelli LA, Cattelin F, Achermann P, et al. Serotonin-2 receptors and human sleep: effect of a selective antagonist on EEG power spectra. Neuropsychopharmacology. 1999. https://doi.org/S0893-133X(99) 00052-4 [pii]/r10.1016/S0893-133X(99)00052-4

52. Von Rotz R, Kometer M, Dornbierer D, Gertsch J, Salomé Gachet M, Vollenweider FX, et al. Neuronal oscillations and synchronicity associated with gamma- hydroxybutyrate during resting-state in healthy male volunteers. https://doi.org/ 10.1007/s00213-017-4603-z

53. Crunelli V, Leresche N. A role for GABABreceptors in excitation and inhibition of thalamocortical cells. Trends Neurosci. 1991.

54. Williams SR, Turner JP, Crunelli V. Gamma-hydroxybutyrate promotes oscillatory activity of rat and cat thalamocortical neurons by a tonic GABAB receptormediated hyperpolarization. Neuroscience. 1995. https://doi.org/10.1016/03064522(94)00604-4

55. Xie X, Smart TG. $Y$-Hydroxybutyrate hyperpolarizes hippocampal neurones by activating GABAB receptors. Eur J Pharmacol. 1992. https://doi.org/10.1016/00142999(92)90347-7

56. Maddock RJ, Garrett AS, Buonocore, MH. Remembering familiar people: the posterior cingulate cortex and autobiographical memory retrieval. Neuroscience. 2001. https://doi.org/10.1016/S0306-4522(01)00108-7

57. Born J, Wagner U. Sleep, hormones, and memory. Obstet Gynecol Clin North Am. 2009. https://doi.org/10.1016/j.ogc.2009.10.001

58. Köhler S, Black SE, Sinden M, Szekely C, Kidron D, Parker JL, et al. Memory impairments associated with hippocampal versus parahippocampal-gyrus atrophy: an MR volumetry study in Alzheimer's disease. Neuropsychologia. 1998. https://doi.org/10.1016/S0028-3932(98)00017-7

59. Leech R, Sharp DJ. The role of the posterior cingulate cortex in cognition and disease. Brain . 2014. https://doi.org/10.1093/brain/awt162

60. Mander BA, Rao V, Lu B, Saletin JM, Lindquist JR, Ancoli-Israel S, et al. Prefrontal atrophy, disrupted NREM slow waves and impaired hippocampal-dependent memory in aging. Nat Neurosci. 2013. https://doi.org/10.1038/nn.3324

61. Czeisler CA, Weitzman ED, Moore-Ede MC, Zimmerman JC, Knauer RS. Human sleep: Its duration and organization depend on its circadian phase. Science. 1980. https://doi.org/10.1126/science.7434029

62. Dijk D, Czeisler C. Contribution of the circadian pacemaker and the sleep homeostat to sleep propensity, sleep structure, electroencephalographic slow waves, and sleep spindle activity in humans. J Neurosci. 1995. https://doi.org/ 10.1523/JNEUROSCI.15-05-03526.1995

63. Brunner DP, Dijk DJ, Tobler I, Borbély AA. Effect of partial sleep deprivation on sleep stages and EEG power spectra: evidence for non-REM and REM sleep homeostasis. Electroencephalogr Clin Neurophysiol. 1990. https://doi.org/ 10.1016/0013-4694(90)90136-8

64. Brancucci A, Berretta N, Mercuri NB, Francesconi W. Gamma-hydroxybutyrate and ethanol depress spontaneous excitatory postsynaptic currents in dopaminergic neurons of the substantia nigra. Brain Res. 2004;997:62-66. https://doi.org/ 10.1016/j.brainres.2003.10.046

65. Cruz HG, Ivanova T, Lunn M-L, Stoffel M, Slesinger PA, Lüscher C. Bi-directional effects of GABAB receptor agonists on the mesolimbic dopamine system. Nat Neurosci. 2004;7:153-159. https://doi.org/10.1038/nn1181

66. Hechler V, Gobaille S, Bourguignon J-J, Maitre, M. Extracellular events induced by Y-hydroxybutyrate in striatum: a microdialysis study. J Neurochem. 1991. https:// doi.org/10.1111/j.1471-4159.1991.tb02012.x

67. Kuschinsky W, Suda S, Sokoloff L. Influence of gamma-hydroxybutyrate on the relationship between local cerebral glucose utilization and local cerebral blood flow in the rat brain. J Cereb Blood Flow Metab. 1985;5:58-64.

68. Labouèbe $G$, Lomazzi $M$, Cruz HG, Creton $C$, Luján R, Li M, et al. RGS2 modulates coupling between GABAB receptors and GIRK channels in dopamine neurons of the ventral tegmental area. Nat Neurosci. 2007;10:1559-1568. https://doi.org/ $10.1038 / \mathrm{nn} 2006$

69. Fritz BA, Kalarickal PL, Maybrier HR, Muench MR, Dearth D, Chen Y, et al. Intraoperative electroencephalogram suppression predicts postoperative delirium. Anesth Analg. 2016. https://doi.org/10.1213/ANE.0000000000000989

70. Gehring WJ, Coles MG, Meyer DE, Donchin E. A brain potential manifestation of error-related processing. Electroencephalogr Clin Neurophysiol Suppl. 1995;44:261-72. http://www.ncbi.nlm.nih.gov/pubmed/7649032 Retrieved from

71. Mamelak M. Alzheimer's disease, oxidative stress and gammahydroxybutyrate. Neurobiol Aging. 2007. https://doi.org/10.1016/j.neurobiolaging.2006.06.008

72. Patat A, Trocherie S, Thebault JJ, Rosenzweig P, Dubruc C, Bianchetti G, et al. EEG profile of intravenous zolpidem in healthy volunteers. Psychopharmacology. 1994. https://doi.org/10.1007/BF02245455

73. Soehle M, Dittmann A, Ellerkmann RK, Baumgarten G, Putensen C, Guenther U. Intraoperative burst suppression is associated with postoperative delirium following cardiac surgery: a prospective, observational study. BMC Anesthesiol. 2015. https://doi.org/10.1186/s12871-015-0051-7

74. Van Lier H, Drinkenburg WHIM, Van Eeten YJW, Coenen, AML. Effects of diazepam and zolpidem on EEG beta frequencies are behavior-specific in rats. Neuropharmacology. 2004. https://doi.org/10.1016/j.neuropharm.2004.03.017 